\title{
Situation et perspectives de la pisciculture dans le monde : consommation et production
}

\author{
Bernard Chevassus-au-Louis ${ }^{1}$ \\ Jérôme Lazard ${ }^{2}$ \\ ${ }^{1}$ Laboratoire de génétique des poissons \\ Inra \\ 78350 Jouy-en-Josas \\ France \\ <bernard.chevassus@jouy.inra.fr> \\ ${ }^{2}$ Cirad \\ UR « Aquaculture et gestion des ressources \\ aquatiques" \\ TA B-20/01 \\ Avenue Agropolis \\ 34398 Montpellier cedex 5 \\ France \\ <jerome.lazard@cirad.fr>
}

\begin{abstract}
Résumé
Au cours des 30 dernières années, la consommation mondiale de protéines animales, relativement stable dans les pays développés, a plus que doublé dans les pays en développement. Cette croissance résulte, principalement, des productions de volailles, mais les produits aquatiques ont également apporté une contribution notable (passage de 6,3 à 13,8 kg par personne et par an), similaire à celle des porcins et très supérieure à celle des ruminants. Cette contribution des produits aquatiques résulte des pêcheries et, plus récemment, de la pisciculture. Le cas des pêcheries minotières fait l'objet d'une analyse spécifique du fait de l'utilisation croissante de ses produits par la pisciculture. La pisciculture, qui représente, depuis 1980, le plus fort taux de croissance de toutes les productions animales, est encore principalement basée sur des productions traditionnelles de poissons d'eau douce et de mollusques en zone côtière. Les différentes prospectives réalisées au début des années 2000 amènent à distinguer nettement la situation des pêcheries où le réexamen à la baisse des captures chinoises, la surexploitation de nombreux stocks et l'échec des politiques de restauration de certains stocks amènent à envisager des scénarios d'érosion progressive des captures totales, tant minotières qu'alimentaires. Dans le domaine de la pisciculture, la dynamique actuelle devrait, en revanche, se poursuivre, avec des taux de croissance sans doute plus modérés. La résultante de ces deux dynamiques devrait aboutir, à échéance 2020, à des contributions équivalentes des pêches (hors pêches minotières) et de l'aquaculture (hors algues) à l'alimentation mondiale, leur contribution totale par habitant n'augmentant pas sensiblement par rapport au niveau actuel.
\end{abstract}

Mots clés : consommation alimentaire ; pêches ; pisciculture ; prospective.

Thèmes : productions animales ; pêche et aquaculture ; ressources naturelles et environnement.

\section{Abstract \\ Current situation and prospects for international fish farming: Consumption and production}

Over the last thirty years, the world consumption of animal protein, although relatively stable in a number of developed countries, has more than doubled in developing countries. This increase is due mainly to poultry farming. However, aquatic products have also strongly contributed to this growth in a similar proportion to pig farming and in a much higher proportion than ruminant farming. This significant contribution from aquatic products is related to fishing areas and, more recently, to fish farming. A specific analysis is being conducted on industrial fishing due to increasing use of its products in fish farming. Since 1980, fish farming has accounted for the highest growth rate of all animal production. Currently, fish farming is based primarily on traditional freshwater fish farming and mollusc farming in coastal areas. Following the prospective carried out at the beginning of the 2000s, scenarios now call for progressive reduction of total captures of both feed and food fishing. However, as regards fish farming, the current trend should continue but at growth rates which will most likely be lower. The result of these two trends should mean that by 2020 , foodfish from fishing and aquaculture (excluding seaweed production) should contribute equally to world food supply thus entailing no significant change in total contribution per capita with respect to the current levels.

Key words: fisheries; fish farming; food consumption; consumption; prospective.

Subjects: animal productions; fishing and aquaculture; natural resources and environment. 
L

a situation et l'évolution des productions animales de l'aquaculture (poissons, mollusques, crustacés) ne peuvent être étudiées indépendamment des productions animales terrestres ni de celles issues de la pêche avec lesquelles elles se trouvent directement en concurrence sur l'un et/ou l'autre des deux marchés : celui de la consommation et celui des aliments d'élevage.

Cet article a pour objet de montrer que la spectaculaire progression de l'aquaculture ("révolution bleue") depuis 1980, avec un taux de croissance ayant atteint $12 \%$ par an durant certaines périodes, vient à point nommé pour compenser une pêche dont le tonnage des débarquements stagne depuis le milieu des années 1990 et dont rien n'indique que la situation s'améliore à l'avenir (les prospectives prévoient même leur diminution). Cette révolution bleue, encore largement basée sur des productions traditionnelles, doit aujourd'hui faire face à des tendances ayant pour nom, par exemple, intensification, diversification vers des espèces à plus forte valeur marchande, toutes deux fortement consommatrices de matières premières liées directement à la pêche (farine et huile de poisson).

\section{Évolutions}

\section{de la production/ consommation de produits aquatiques animaux de 1968 à 1998}

\section{Évolution des différentes productions}

La production mondiale de produits animaux a, en moyenne, doublé de 1968 à 1998 , mais ce chiffre recouvre des situations très différentes (figure 1) : les produits issus des ruminants (lait et viande de bovins, ovins et caprins) ont progressé modérément (+50\% environ) et leur consommation par habitant a fléchi dans les pays développés et n'a que faiblement progressé dans les pays en développement; à l'inverse, la viande de volailles a connu un développement spectaculaire. Entre ces deux extrêmes, les œufs, la viande de porc et les produits aquati- ques constituent un ensemble intermédiaire, assez homogène en termes de progression.

Les produits aquatiques jouent un rôle beaucoup plus important dans les pays en développement, où leur apport net (consommation humaine directe) est passé de 6,3 à 13,8 kg par personne et par an pendant cette période.

En termes de contribution de ces différents produits à l'augmentation de la ration alimentaire des PED, la part des ruminants a été de $20 \%$ (dont $15 \%$ pour le lait), celle des monogastriques de $58 \%$, celle des produits aquatiques de $22 \%$.

On observe également que ces produits aquatiques sont répartis de manière plus "égalitaire " que les autres productions animales: la ration des habitants des PED en produits aquatiques est d'environ $60 \%$ de celle des habitants des pays développés, alors que les valeurs sont respectivement de 26, 29 et $37 \%$ pour les viandes de bovins, de porcins et de volailles.

\section{Contribution des produits aquatiques}

Dans cet article, nous ne traiterons pas des productions d'algues à des fins alimentaires ou non alimentaires. Ces productions représentaient, en 2002, environ 13 millions de tonnes (1,2 MT de pêche et
11,6 MT d'aquaculture, soit environ $20 \%$ en volume et $10 \%$ en valeur de la production aquacole mondiale). Elle est assurée à $90 \%$ par trois pays, la Chine, le Japon et les Philippines.

La contribution des produits aquatiques résulte de deux phénomènes distincts : le développement des pêches industrielles depuis les années 1950 et, plus récemment, l'essor de l'aquaculture (figure 2). Si l'on se limite aux produits destinés à l'alimentation humaine directe, le total de ces deux activités a franchi en 2002 la barre symbolique des 100 millions de tonnes, soit une multiplication par six depuis 1950. L'essentiel des données présentées ciaprès est issu de la base statistique FAO spécialisée Fisheries Global Information System (FIGIS : www.fao.org/figis/servlet/), dans les rapports de synthèse bisannuels The State of World Fisheries and Aquaculture (SOFIA) et dans la revue de la situation de l'aquaculture mondiale établie pour l'an 2000 (FAO, 2003).

Bien que cet article soit consacré à l'aquaculture, et plus spécifiquement à la pisciculture, les très nombreuses interactions entre les pêches et l'aquaculture nous amènent à évoquer brièvement les données relatives aux pêches.

\section{Pêcheries}

L'essor des pêches a commencé au début du $x^{e}$ siècle et, après une pause lors de la

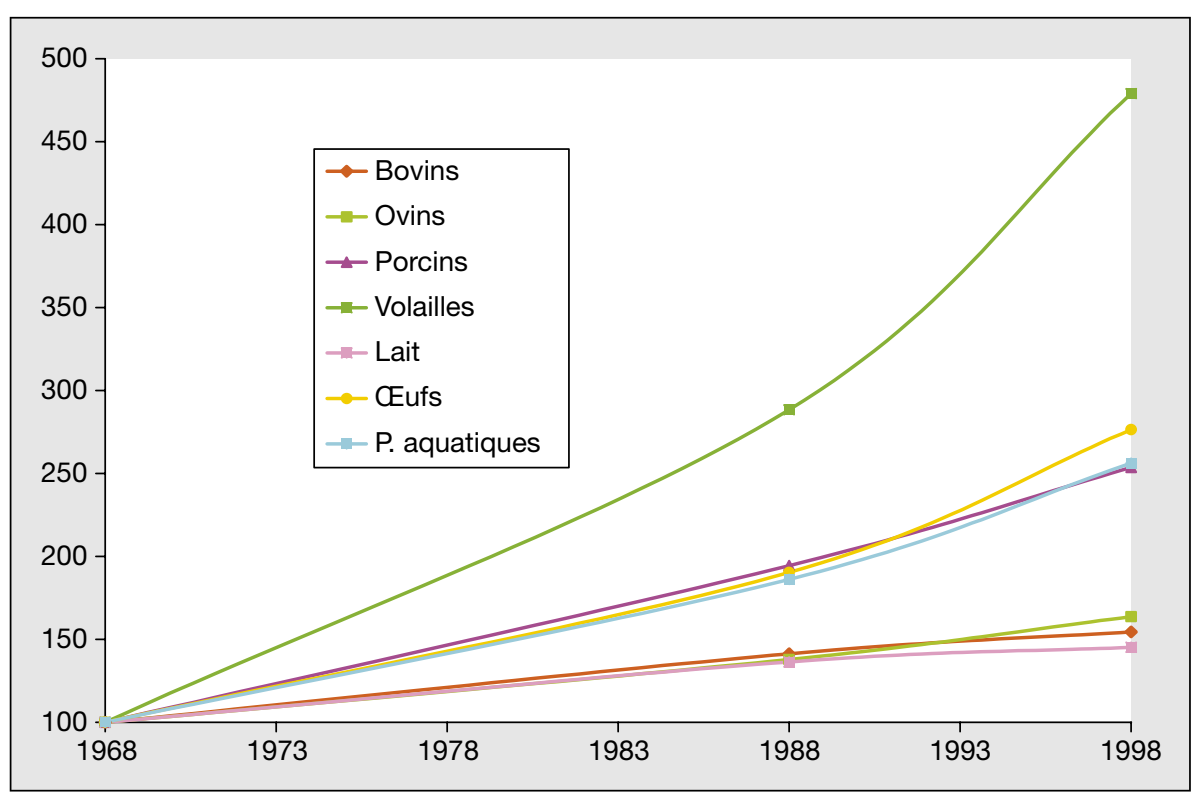

Figure 1. Évolution de la production mondiale de produits animaux 1968 à 1998 (base $100=1968$ ).

Figure 1. Variation in world production of animal products between 1968 and 1998 (base $100=1968$ ). Source : FAOSTAT. 


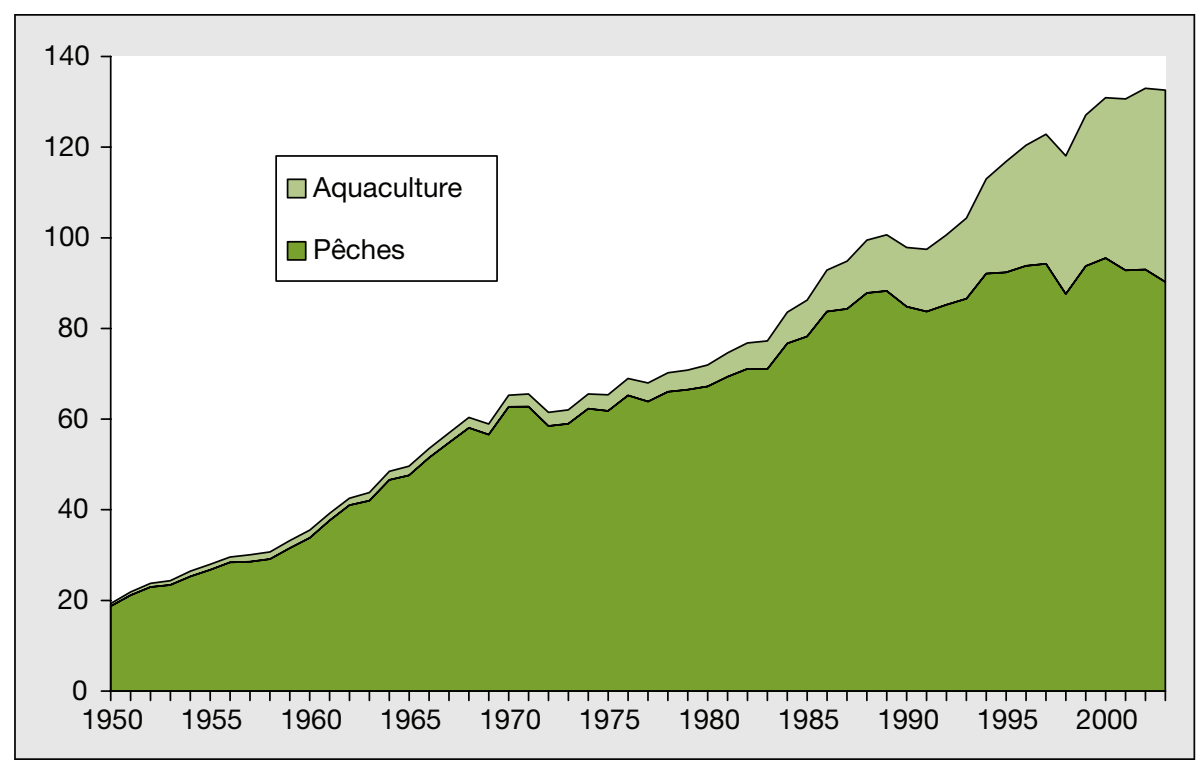

Figure 2. Production des pêches (y compris pêches minotières) et de l'aquaculture mondiale (hors algues) de 1950 à 2003 (millions de tonnes).

Figure 2. World fisheries (including fisheries destined for reduction) and aquaculture (excluding aquatic plants) production between 1950 and 2003 (millions of tons).

Source : FAOSTAT.

Seconde Guerre mondiale, a repris à partir des années 1950: de 1950 à 2000, le total des captures a été multiplié par cinq. La contribution des pêcheries par habitant a cru fortement jusque dans les années 1970, avec un doublement par rapport à 1950, pour ensuite se stabiliser. Il s'agit, ici, d'une contribution brute, en équivalent poids frais, comprenant les usages non destinés à l'alimentation humaine directe, y compris les usages non alimentaires. On parlera de contribution nette pour désigner la part dédiée directement à l'alimentation humaine.

Environ $90 \%$ de ces captures sont réalisées en mer, cette proportion étant stable depuis les années 1960. Cependant, la situation est contrastée selon les continents: les pêcheries européenne et nord-américaine sont essentiellement marines alors que les pêches dans les eaux intérieures représentaient, en 2000, $29 \%$ des pêches africaines et $12 \%$ des pêcheries asiatiques.

L'importance de ces pêches intérieures en Afrique et en Asie du Sud-Est ne doit pas être sous-estimée : dans des pays comme le Burundi, le Congo, le Malawi, la Tanzanie, le Laos les produits aquatiques représentent de 25 à $40 \%$ de la ration de protéines animales et émanent pour l'essentiel de ces pêches. Un cas extrême est celui du Cambodge : en 2003, 52 \% de

\footnotetext{
a Delgado et al., 2003.

b Fishmeal Information Network.

c IFFO, 2007.

d Pike, 2005.
}

un million de tonnes d'huiles en 2006 (IFFO, 2007). Ces productions de farines et d'huiles pour l'alimentation animale représentent l'essentiel (environ 90\%) des utilisations autres que l'alimentation humaine des produits aquatiques. Le reste est constitué d'environ trois millions de tonnes de captures utilisées pour confectionner des aliments "frais " (y compris conserves) pour certains élevages (animaux de compagnie, animaux à fourrures, poissons marins) et pour divers usages industriels.

En termes d'utilisation, cette production était, jusqu'aux années 1990, essentiellement destinée à l'alimentation des porcs et des volailles (tableau 1), et a contribué de manière notable à l'essor de ces productions. Cependant, cette utilisation par les productions animales terrestres a entamé un déclin depuis les années 1990, avec une substitution croissante par les tourteaux de soja, en particulier pour l'alimentation des volailles. La production mondiale de soja a en effet décuplé depuis 1961, et son prix relatif par rapport aux farines de poisson a considérablement baissé au cours des 20 dernières années (figure 3). On observe, à l'inverse, une utilisation croissante par la pisciculture avec, en outre, une tendance à l'augmentation de l'autoconsommation des farines de poisson par les pays producteurs, du fait notamment du développement de la pisciculture au Chili, au Japon et en Norvège : le rapport consommation/production du total des huit premiers producteurs mondiaux de farines est passé de 66 \% en 1996 à 83 \% en 2004.

\section{Pisciculture}

Limitée à un à deux millions de tonnes dans la période 1950-1970, la production mondiale a connu une croissance soutenue à partir des années 1980, pour dépasser en 2003 le cap des 40 millions de ton-

\section{Tableau 1. Utilisation des farines de poisson (en pourcentage du total) par les différentes productions animales.}

Table 1. Use of fishmeal (in percentage of total) by the different animal productions.

\begin{tabular}{lccccccc}
\hline Usage & $\mathbf{1 9 8 4}^{\mathbf{a}}$ & $\mathbf{1 9 8 8}^{\mathbf{a}}$ & $\mathbf{1 9 9 4}^{\mathbf{a}}$ & $\mathbf{2 0 0 0}^{\mathbf{a}}$ & $\mathbf{2 0 0 2}^{\mathbf{b}}$ & $\mathbf{2 0 0 6}^{\mathbf{c}}$ & $\mathbf{2 0 1 2}^{\mathbf{d}}$ \\
\hline Aquaculture & 8 & 10 & 17 & 35 & 39 & 57 & 50 \\
Porcs & & 20 & 20 & 29 & 23 & 22 & 25 \\
Volailles & 92 & 60 & 55 & 24 & 28 & 14 & 15 \\
Autres & & 10 & 8 & 8 & 10 & 7 & 10
\end{tabular}




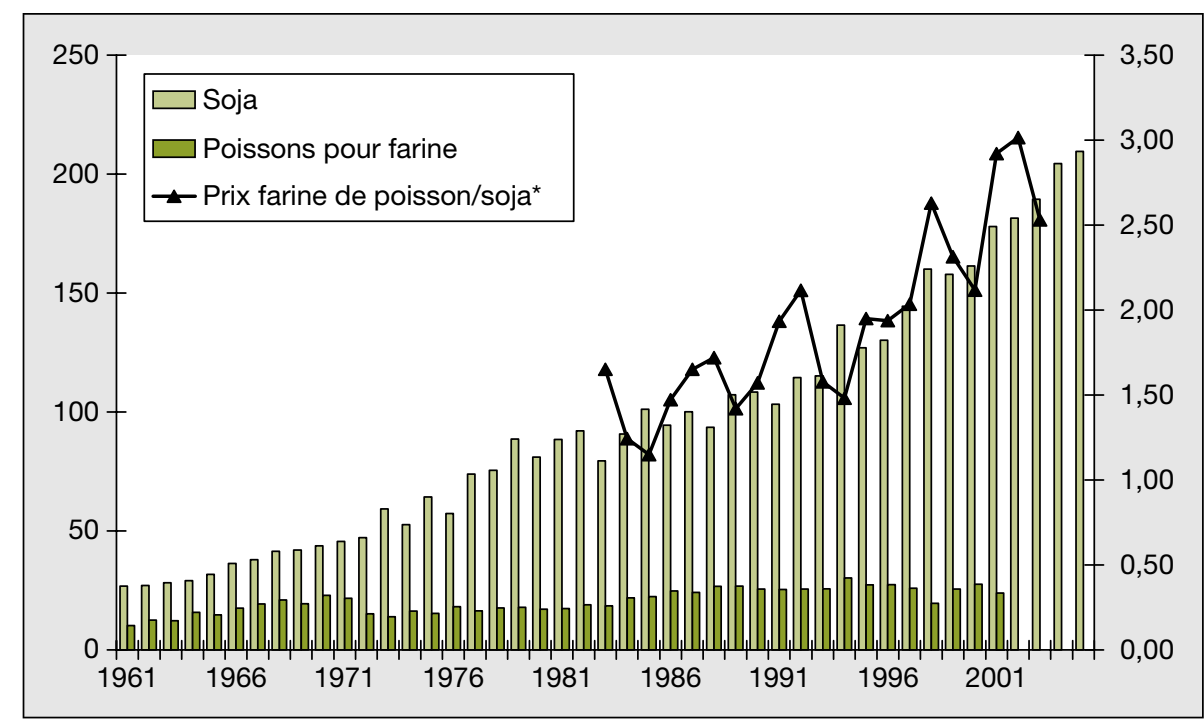

Figure 3. Évolution des productions mondiales de soja et de poissons pour farines depuis 1961 (en millions de tonnes, axe de gauche) et du prix relatif des farines de poisson par rapport au soja* (axe de droite).

Figure 3. Variation in world soya and fish productions for feed meal since 1961 (in millions of tons, left axis) and relative price of fishmeal compared to soya (right axis).

Source : FAOSTAT.

* Nous avons construit cet indice comme le rapport entre le prix à l'exportation des farines de poisson du Chili et du Pérou (les deux premiers exportateurs mondiaux, prix moyen non pondéré) et celui du prix à l'exportation du soja des États-Unis, du Brésil et de I'Argentine (les trois premiers exportateurs mondiaux pendant la période considérée, moyenne non pondérée). nes. Ce taux de croissance est, depuis 1980, le plus élevé de toutes les productions animales (figure 4) et dépasse nettement celui des volailles et des porcins, l'écart étant moins net si l'on exclut les données chinoises. De ce fait, la contribution de l'aquaculture à l'alimentation humaine est passée de moins de $1 \mathrm{~kg} /$ habitant par an dans les années 1970 à plus de $6 \mathrm{~kg}$ aujourd'hui.

Ces chiffres ne tiennent pas compte des activités mixtes où la pisciculture produit des juvéniles destinés au repeuplement et exploités par la pêche. Dans le domaine marin, ces activités, commencées dès la fin du XIX ${ }^{\mathrm{e}}$ siècle, concernent de nombreuses espèces, mais leur efficacité est aujourd'hui controversée (Salvanes, 2001 et FAO, 2004 pour des revues), à l'exception notable des repeuplements japonais en saumon keta (Oncorbynchus keta) qui seraient à l'origine de $90 \%$ des captures en mer de ce pays (environ 200000 tonnes). Dans les eaux continentales, des formes de repeuplement de plans d'eaux temporaires ou permanents se sont développées plus récemment, notamment en Asie et en Amérique du Sud (De Silva et al., 2003), et apparaissent beaucoup plus prometteuses. Elles seraient, par exemple, à l'origine de la production de plus d'un million de tonnes de poissons en Chine.

À l'inverse des productions animales terrestres, les productions aquacoles sont basées sur un très grand nombre d'espè- ces : en 2004, la FAO a recensé plus de 240 espèces animales et végétales élevées (SOFIA, 2006), 25 d'entre elles assurant néanmoins $86,6 \%$ de la production et 15 espèces de poissons assurant, quant à elles, $85 \%$ de la production de la pisciculture mondiale.

L'examen attentif des composantes de ce "boom" aquacole montre :

- qu'il est essentiellement le fait des pays en développement, avec une part prépondérante de l'Asie (92\%) et, en particulier, de la Chine (70 \%). Les pays développés, qui représentaient, en 1973, $42 \%$ de la production aquacole mondiale, n'en représentaient plus que $29 \%$ en 1985 et $9,2 \%$ en 2003 . Au sein des pays développés, l'Europe (ex-URSS incluse) a une part prépondérante, suivie du Japon

- que cette croissance est due, pour l'essentiel, à des productions déjà bien établies dans les années 1970: poissons d'eau douce, principalement en étangs, et mollusques en zones côtières (figure 5). Les trois espèces aquacoles les plus élevées dans le monde, en 2004, étaient d'ailleurs l'huitre japonaise Crassostrea gigas (4,4 millions de tonnes) et la carpe argentée Hypophthalmichtes deux phytoplantonophages, et la "carpe herbivore" Ctenopharyngodon idellus. Les productions, plus récentes, de poissons marins et de crustacés en et des États-Unis ; thys molitrix (4,0 millions de tonnes), tou-

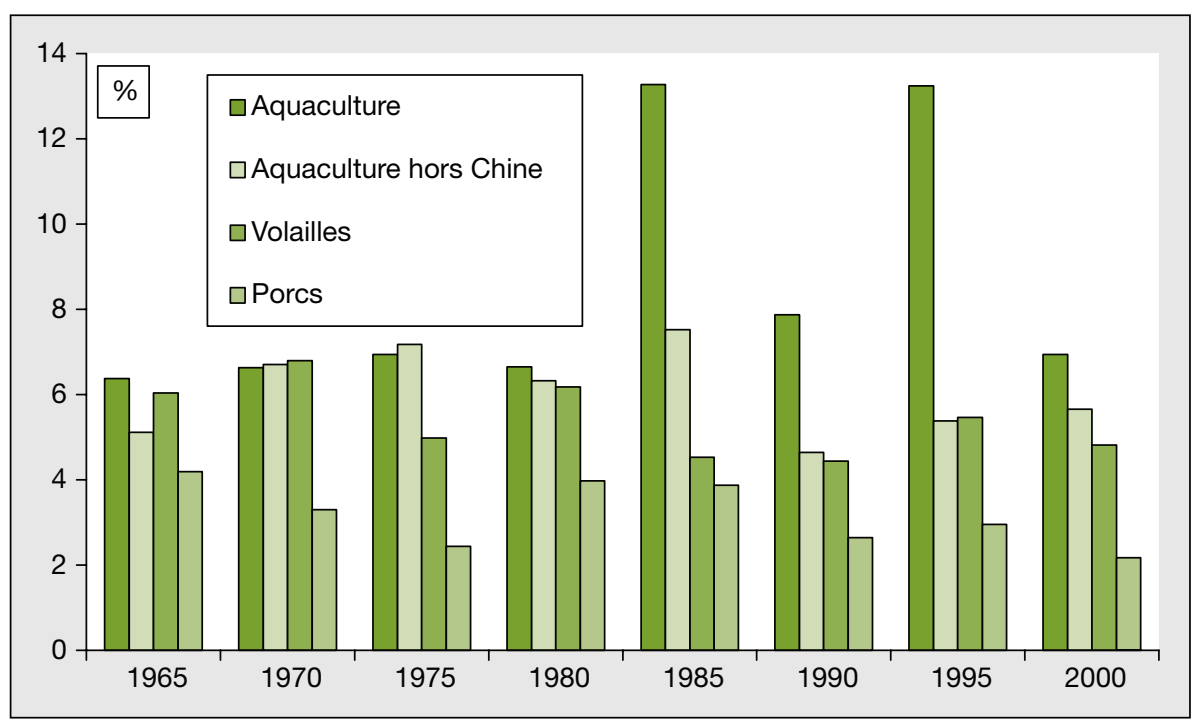

Figure 4. Taux de croissance annuelle en pourcentage des productions mondiales de volailles, de porcs et de produits aquacoles animaux (moyennes quinquennales centrées).

Figure 4. Yearly growth rate in percentage of world poultry, pig and animal aquaculture productions (averages centered over five years).

Source : FAOSTAT. 


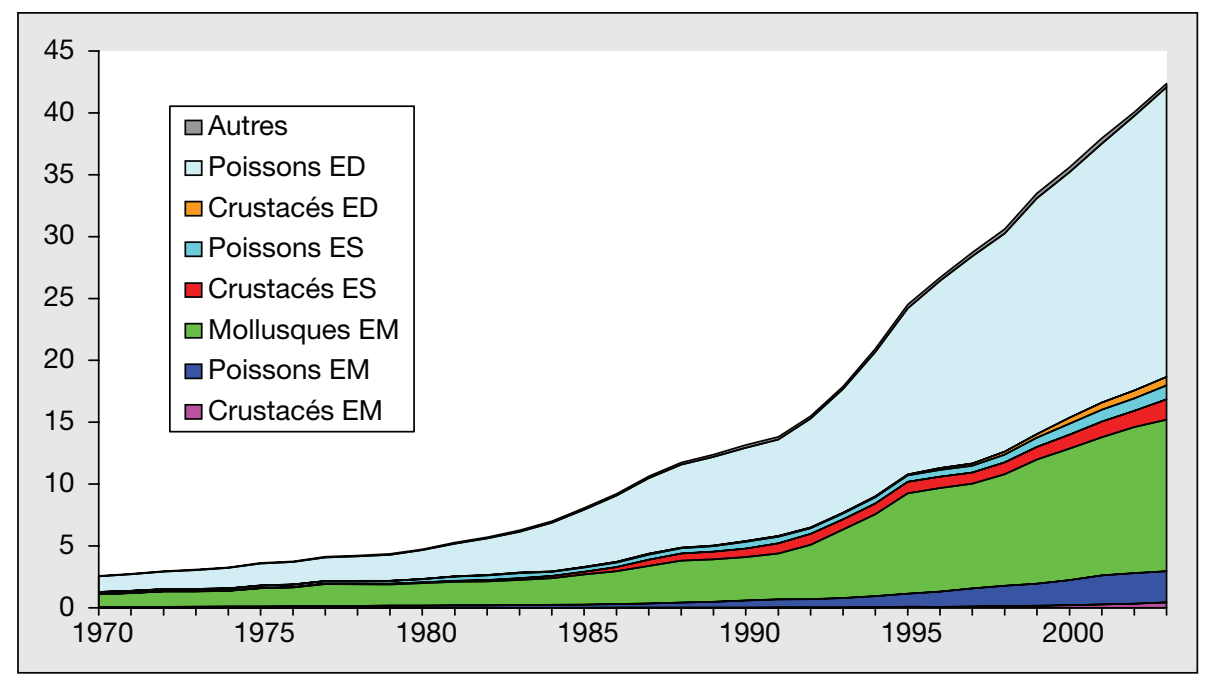

Figure 5. Production aquacole mondiale par groupe d'espèces et par milieu (en millions de tonnes).

Figure 5. World aquaculture production per group of species and per environment (in millions of tons). Source : FAOSTAT

$E D=$ eaux douces $; E S$ = eaux saumâtres $; E M=$ eaux marines.

eaux saumâtres sont encore nettement inférieures en volume, même si leur part en valeur est nettement plus élevée : en 2003 , les productions de poissons marins, de salmonidés et de crustacés représentaient $14 \%$ de la production totale en volume, mais $40 \%$ en valeur. Il existe également une production de crustacés en eau douce, qui représente environ un tiers de la production totale et est basée principalement sur trois espèces, l'écrevisse rouge des marais (Procambarus clarkii), le bouquet géant (Macrobrachium rosenbergii) et, surtout, le crabe chinois de rivière (Eriocher sinensis), dont la production, inexistante en 1998, a dépassé les 400000 tonnes en 2004.

\section{Perspectives de production/ consommation des produits aquatiques}

La figure 6 présente quelques estimations récentes de l'évolution de la consommation. Les études de Ye (1999) et de Wijkström (2003) ont cherché à évaluer la demande potentielle de produits aquatiques à diverses échéances, en se basant uniquement sur les tendances de la productions animales terrestres (Delgado et al., 1999), qui permet des substitutions de consommation via les prix relatifs des produits. Il propose un scénario tendanciel, considéré comme le plus plausible, et plusieurs scénarios alternatifs concernant soit les pêcheries, soit l'aquaculture. C'est cette étude qui est retenue ici.

\section{Pêcheries}

Des différents scénarios envisagés par Delgado et al. (2003), le plus radical (ecological collapse, selon le terme de Jackson et al., 2001) prend en compte les échecs de nombreux modèles de gestion des pêcheries, qui n'avaient pas suffisamment intégré la dégradation des habitats liée aux modes de pêches modernes et ses conséquences sur la dynamique de renouvellement des stocks. Ainsi, Cury et Morand (2005) indiquent que seulement $7 \%$ des stocks qui se sont effondrés ont vu une récupération de leurs effectifs en une génération après l'arrêt de leur exploitation, l'exemple de la morue de l'Atlantique étant le plus emblématique. En effet, compte tenu de la forte fécondité des poissons, on considère que le nombre de reproducteurs n'est généralement pas un facteur limitant pour engendrer, si les capacités d'accueil du milieu sont favorables, une population abondante dès la repris le modèle IMPACT utilisé pour les

consommation et sur l'évolution de la population et du pouvoir d'achat. Ces études fournissent donc des estimations de "demande potentielle" considérables, mais la question de la capacité de la production à satisfaire cette demande n'est pas posée, ce qui limite l'intérêt de telles

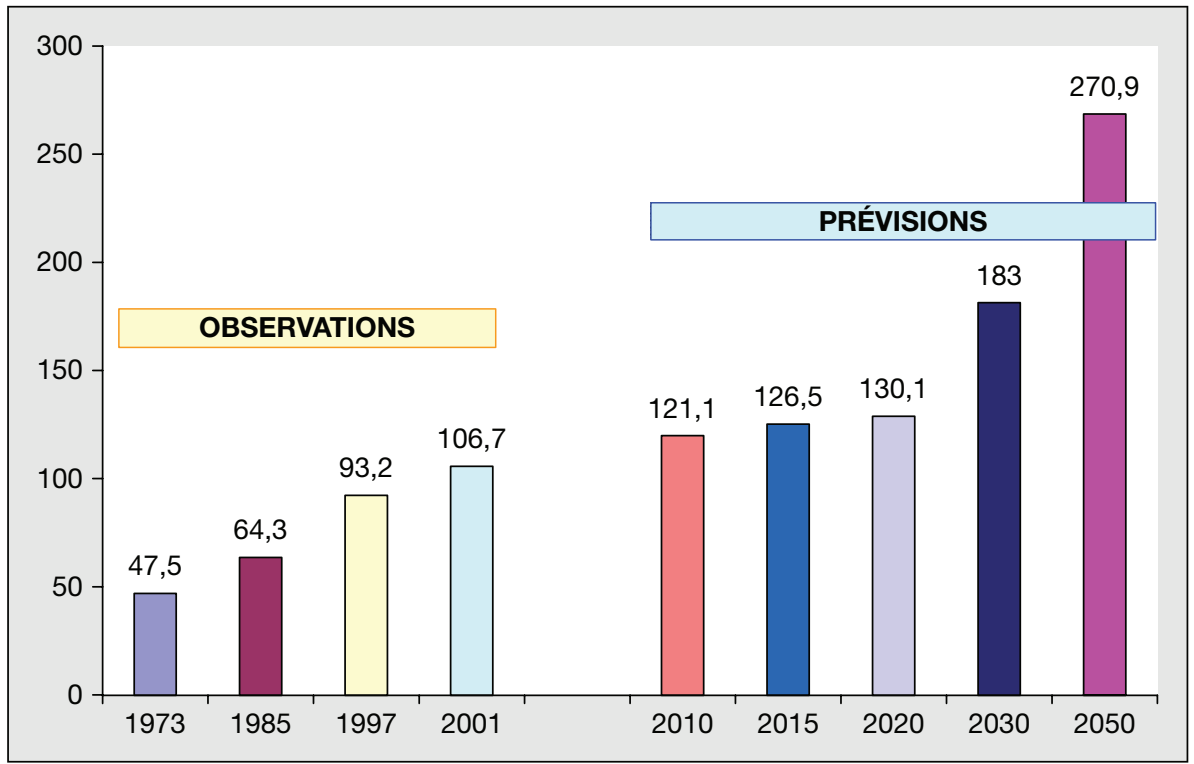

Figure 6. Évolution des productions aquatiques destinées à l'alimentation humaine directe (en millions de tonnes).

Figure 6. Variation in aquaculture production intended for direct human consumption (in millions of tons).

Sources: Observations: FAOSTAT; Prévisions 2010 et 2050 : Wijkström, 2003 ; Prévisions 2015 et 2030 : Ye, 1999 ; Prévision 2020 : Delgado et al., 2003. 
génération suivante. Ce scénario extrapole et amplifie la tendance baissière précédemment évoquée et envisage, par rapport à 1997, une baisse de plus de $15 \%$ des pêcheries mondiales d'ici 2020, ce qui les ramènerait au niveau du début des années 1980.

Cette vision assez pessimiste de l'avenir des pêcheries est de plus en plus partagée. Ainsi, une analyse portant sur les principaux stocks de poissons (SOFIA, 2004) indique qu'environ la moitié d'entre eux est pleinement exploitée, cette proportion étant stable depuis 1974. En revanche, alors qu'à cette date on considérait que $10 \%$ des stocks étaient surexploités ou épuisés, ce chiffre a atteint $24 \%$ en 2003 et, à l'inverse, le taux de stocks dont l'exploitation pouvait être accrue a chuté de 40 à $24 \%$. Il semble donc que les quelques capacités d'exploitation encore disponibles soient au moins compensées par la nécessaire réduction de la pression de pêche sur d'autres stocks.

Dans tous les cas, la contribution nette des pêcheries par habitant régresserait, du fait de l'évolution démographique: de 10,9 kg par an en 1997, elle passerait à $10 \mathrm{~kg}$ dans le scénario tendanciel, à 9,1 kg dans le scénario incluant la correction des pêcheries chinoises, et à $7 \mathrm{~kg}$ dans le scénario d'effondrement écologique. La tendance observée de 1997 à 2003 s'inscrit plutôt dans les scénarios pessimistes: depuis 1995, les captures pour l'alimentation humaine directe plafonnent autour de 61 millions de tonnes (SOFIA, 2002 et 2004).

Pour ce qui concerne les farines de poisson, la tendance depuis 1997 est à la baisse (10\% en moyenne sur la période 1998-2003 par rapport à 1988-1997) et la principale menace qui semble planer sur l'avenir de ces stocks est constituée par les conséquences éventuelles du réchauffement climatique sur la réduction de l'upwelling sud-américain.

En outre, la tendance à une utilisation croissante des farines et des huiles de poisson par l'aquaculture devrait se poursuivre: en 2006, les consommations de farine de poisson $(57 \%)$ et d'huile de poisson (87\%) pour l'aquaculture égalaient ou dépassaient déjà le niveau des prévisions établies pour 2012 : 50 et $88 \%$ respectivement! (Pike, 2005). Une étude de la FAO (New et Wijkström, 2002) considère que, même en intégrant des hypothèses réalistes sur une augmentation de l'efficacité alimentaire et une réduction du taux d'incorporation des farines de poisson dans les aliments com- posés, l'extrapolation des tendances actuelles de l'aquaculture conduirait à utiliser $70 \%$ des farines en 2015 et $159 \%$ en 2030 pour cette activité, d'où l'expression de fishmeal trap utilisée par les auteurs.

Enfin, cette ressource, longtemps monopolisée par un segment aujourd'hui restreint des productions aquacoles (tableau 2), essentiellement les productions intensives d'espèces carnivores (salmonidés, poissons marins et crevettes) voit son utilisation par des espèces à chaîne alimentaire courte croître rapidement au nom de l'intensification des systèmes d'élevage ( $\mathrm{Li}, 2002)$, les rendements piscicoles en étangs et en réservoir ont été multipliés par un facteur de 6,4 et 10,2 entre 1980 et 2000, respectivement en étangs et en réservoir).

En 2003, par exemple, les quantités totales de farine de poisson utilisées pour l'élevage du saumon (573000t) équivalaient approximativement à celles utilisées pour l'élevage des carpes et tilapias (517000 t).

\section{Aquaculture}

Dans le scénario tendanciel de l'International Food Policy Research Institute (IFPRI ; Delgado et al., 2003), l'aquaculture poursuit sa progression à un rythme plus modéré, en particulier en Asie, avec un taux moyen de 2,6\% par an dans les PED et de 2,1\% dans les pays développés. Elle atteindrait en 2020 une production totale de 53,5 millions de tonnes, ce qui correspondrait à une augmentation de la production mondiale de $87 \%$ par rapport à 1997. Les pays asiatiques, et en particulier la Chine, demeureraient de très loin les producteurs les plus importants (tableau 3).

Le cas de l'aquaculture chinoise mérite, du fait de son importance (70\% de la production mondiale), un commentaire spécifique. La FAO considère, en effet, que les problèmes statistiques affectant les pêcheries concernent également l'aquaculture de ce pays (SOFIA, 2004) et analyse désormais ces deux secteurs en distinguant systématiquement la Chine et le reste du monde. Alors que, suite à ces débats, les déclarations chinoises relatives aux pêcheries se sont stabilisées depuis 1998, les déclarations relatives à l'aquaculture continuent à afficher des progressions notables. Néanmoins, bien que la Chine ait annoncé de 1985 à 1997 des taux de croissance annuelle moyens impressionnants de plus de $15 \%$, soit presque le double de la moyenne des autres pays asiatiques $(8,6 \%)$, le taux de ce pays pour la période 1998-2005 (6,6\%) s'est rapproché nettement de celui du reste de l'Asie et lui est même maintenant inférieur. On peut donc considérer comme réaliste l'hypothèse de Delgado et al. (2003) d'un fort tassement et d'un alignement des taux chinois, même si la base de départ demeure sujette à caution. Ce scénario tendanciel est encadré par deux scénarios alternatifs assez simples - supposant une croissance de l'aquaculture d'environ $50 \%$ inférieure ou supérieure à la prévision - qui conduisent à

\section{Tableau 2. Utilisation des farines et huiles de poisson par les principales espèces aquacoles objets d'élevage avec alimentation artificielle et parts relatives de ces productions en 2005 (en volume ; Tacon et al., 2006).}

Table 2. Use of fishmeal and oil by the various aquaculture fed species and relative production proportion in 2005 (in volume; Tacon et al., 2006).

\begin{tabular}{lccc}
\hline Espèces & $\begin{array}{c}\text { Farines } \\
\text { de poisson } \\
(\mathbf{\%})\end{array}$ & $\begin{array}{c}\text { Huiles } \\
\text { de poisson } \\
\mathbf{( \% )}\end{array}$ & $\begin{array}{c}\text { Production } \\
\text { en milliers de tonnes } \\
\text { et en pourcentage }\end{array}$ \\
\hline Crustacés & 31,0 & 14,6 & $2986(14,2)$ \\
Poissons marins & 22,6 & 18,2 & $1332(6,3)$ \\
Saumons & 18,7 & 30,0 & $1388(6,6)$ \\
Truites & 4,8 & 12,7 & $588(2,8)$ \\
Anguilles & 5,4 & 2,0 & $237(1,1)$ \\
Chanos & 1,0 & 1,0 & $597(2,8)$ \\
Tilapia & 2,1 & 3,3 & $2030(9,7)$ \\
Carpes & 13,7 & 16,5 & $11222(53,4)$ \\
Total & 100,0 & 100,0 & $21031(100,0)$ \\
\hline
\end{tabular}


Tableau 3. Taux de croissance moyen (TC en pourcentage par an) de l'aquaculture prévus par I'IFPRI de 1997 à 2020 et taux observés avant 1997 et de 1997 à 2005. Données par pays.

Table 3. Average growth rate (GR in percentage per year) predicted for aquaculture by IFPRI between 1997 and 2020 and rates observed prior to 1997 and between 1997 and 2003; data per country.

\begin{tabular}{|c|c|c|c|c|c|c|}
\hline & $\begin{array}{c}1997 \\
\left(10^{3} t\right)\end{array}$ & $\begin{array}{c}\text { TC } \\
1985-1997\end{array}$ & $\begin{array}{c}\text { TC } \\
1997-2020\end{array}$ & $\begin{array}{l}\text { Prévision } \\
2020\left(10^{3} t\right)\end{array}$ & $\begin{array}{c}2005 \\
\left(10^{3} t\right)\end{array}$ & $\begin{array}{c}\text { TC } \\
1997-2005\end{array}$ \\
\hline Chine & 19487 & 15,6 & 2,6 & 35122 & 32414 & 6,6 \\
\hline Asie du Sud-Est & 4647 & 8,6 & 3,7 & 10672 & 9431 & 9,2 \\
\hline Ouest asiatique et Afrique & 230 & 9,4 & 4,0 & 566 & 924 & 18,9 \\
\hline Amérique latine & 656 & 19,4 & 3,5 & 1452 & 1377 & 9,7 \\
\hline Total PED & 25431 & 13,3 & 2,8 & 48455 & 44473 & 7,2 \\
\hline Japon & 791 & 1,6 & 1,2 & 1044 & 746 & $-0,8$ \\
\hline États-Unis & 427 & 1,9 & 2,7 & 796 & 471 & 1,2 \\
\hline Europe 15 & 1226 & 3,3 & 2,1 & 1972 & 1207 & $-0,2$ \\
\hline Europe de I'Est + ex-URSS & 189 & $-6,4$ & 0,4 & 206 & 269 & 4,5 \\
\hline Autres & 584 & 17,8 & 2,9 & 1125 & 1004 & 7,0 \\
\hline Total DEV & 3217 & 2,7 & 2,1 & 5143 & 3696 & 1,7 \\
\hline
\end{tabular}

Source: d'après Delgado et al. 2003 ; FAOSTAT.

DEV : pays développés ; PED : pays en voie de développement.

des progressions, de 1997-2020, allant de 43 à $142 \%$, soit une production en 2020 comprise entre 41 et 69 millions de tonnes. Enfin, un scénario de forte augmentation de l'efficacité de transformation des aliments, liée à des innovations biotechniques est envisagé, mais n'a que peu de conséquences sur les volumes de production. Dans ce cas, la tendance à l'augmentation de l'efficacité d'utilisation des farines et huiles animales, estimée à $1 \%$ par an dans le scénario tendanciel, est portée à $2 \%$. Ce scénario ne majore que de $1 \%$ environ la production aquacole totale.

L'examen des tendances observées de 1997 à 2005 révèle une dynamique forte (taux de croissance annuel moyen de 6,7\%), supérieure au scénario le plus optimiste. La production totale a atteint en 200548 millions de tonnes, soit $90 \%$ de l'objectif moyen identifié par l'IFPRI pour 2020. Une étude récente (Brugère et Ridler, 2004), basée sur le cumul des prévisions des principaux pays aquacoles, estime également que le taux de croissance pourrait se maintenir à 4,5\% par an d'ici 2020, conduisant à une production totale comprise entre 85 et 96 millions de tonnes (contre 69 dans le scénario le plus favorable de l'IFPRI).

On observe, cependant, des différences notables en groupes de pays (tableau 3). Le taux de croissance demeure globalement beaucoup plus élevé dans les pays en développement. De ce fait, leur part dans la production totale mondiale, qui était de $71 \%$ en 1985 et de $89 \%$ en
1997, a atteint $92 \%$ en 2005. Outre l'Asie et l'Amérique latine qui confirment leur dynamisme, on observe un début de développement des productions au Moyen-Orient (Iran et Turquie essentiellement) et en Afrique. Dans ce dernier cas, ce développement ne concerne que trois pays (Égypte, Ouganda et Nigeria, qui regroupent à eux trois $94 \%$ de la production africaine, avec $83 \%$ pour la seule Égypte) et s'appuie sur des productions de poissons d'eau douce (tilapias, mais aussi divers poissons chats et cyprinidés). À l'inverse, le tassement de la croissance des productions dans les pays développés apparaît plus net que prévu, avec même des régressions (Japon, Europe des 15).
Ainsi, un groupe de six pays européens d'aquaculture "traditionnelle" (Italie, Espagne, France, Pays-Bas, Allemagne, Danemark) qui représentaient, en 1985, $61 \%$ de la production européenne totale (incluant la Norvège et l'ex-URSS) ont vu leur production baisser de $12 \%$ de 1997 à 2005 et ne représentent plus que $38 \%$ de cette production européenne. En revanche, les pays développant les élevages marins de saumons (Norvège, Irlande, Royaume-Uni) ou de bars et daurades (Grèce) se confortent: ces quatre pays ont augmenté de $71 \%$ leur production pendant la même période et représentent maintenant $41 \%$ de la production européenne totale. Enfin, on observe égale-

\section{Tableau 4. Taux de croissance moyen (TC en pourcentage par an) de I'aquaculture prévus par I'IFPRI de 1997 à 2020 et taux observés avant 1997 et de 1997 à 2005. Données par produits.}

Table 4. Average growth rate (GR in percentage per year) predicted for aquaculture by IFPRI between 1997 and 2020 and rates observed prior to 1997 and between 1997 and 2003. Data per product.

\begin{tabular}{lrccccc}
\hline & $\begin{array}{c}\mathbf{1 9 9 7} \\
\left(\mathbf{1 0}^{\mathbf{3}} \mathbf{t}\right)\end{array}$ & $\begin{array}{c}\text { TC } \\
\mathbf{1 9 8 5}-\mathbf{1 9 9 7}\end{array}$ & $\begin{array}{c}\text { TC } \\
\mathbf{1 9 9 7 - 2 0 2 0}\end{array}$ & $\begin{array}{c}\text { Prévision } \\
\mathbf{2 0 2 0}\left(\mathbf{1 0}^{\mathbf{3}} \mathbf{t}\right)\end{array}$ & $\begin{array}{c}\mathbf{2 0 0 5} \\
\left.\mathbf{( 1 0}^{\mathbf{3}} \mathbf{t}\right)\end{array}$ & $\begin{array}{c}\text { TC } \\
\mathbf{1 9 9 7 - 2 0 0 5}\end{array}$ \\
\hline Poissons FV & 17193 & 10,9 & 2,8 & 32370 & 30301 & 6,3 \\
Poissons HV & 1392 & 13,4 & 2,6 & 2519 & & \\
Crustacés & 1307 & 13,3 & 3,2 & 2719 & 3961 & 14,9 \\
Mollusques & 8755 & 11,1 & 2,7 & 15989 & 13448 & 5,5 \\
Total & $\mathbf{2 8} \mathbf{6 4 7}$ & & & $\mathbf{5 3 5 9 7}$ & $\mathbf{4 8} \mathbf{1 6 9}$ & $\mathbf{6 , 7}$
\end{tabular}

FV : faible valeur; HV : haute valeur. 
ment un redressement de la production des pays de l'Est de l'Europe, lié principalement au redémarrage des productions russes, qui retrouvent le niveau des années 1990 après une forte chute dans la période 1995-2000.

En ce qui concerne les principaux produits (tableau 4), le tassement du taux de croissance serait légèrement plus marqué pour les poissons de haute valeur marchande, du fait notamment de leur dépendance vis-à-vis des farines et huiles de poisson. Les principales augmentations de production seront celles des poissons de faible valeur (production d'eau douce en étangs) et de mollusques, ce qui, compte tenu des profils de production des différents pays, confortera la prédominance asiatique. Une croissance soutenue est également prévue pour les productions de crustacés et la période 1997-2003 confirme le dynamisme de cette activité.

Une conséquence importante de cette forte dynamique de l'aquaculture est le fait que sa contribution à la production totale de produits aquatiques pour l'alimentation humaine (pêches minotières exclues) dépassera en volume le cap symbolique de $50 \%$ pour les poissons de faible valeur d'ici 2020, après l'avoir fait pour les mollusques dans les années 1990 (figure 7). En revanche, et contrairement à une vision répandue, les poissons de haute valeur continueront à être fournis essentiellement par la pêche, salmonidés, bars et daurades constituant plutôt des exceptions dans ce panorama.

\section{Échanges de produits aquatiques}

L'analyse des échanges de produits aquaet pays en développement de 1985 à 2020 fait apparaître une tendance croissante à l'autoconsommation au sein de ces deux blocs: les exportations de poissons de faible valeur des pays développés seront en diminution et, à l'inverse, les productions de crustacés et de poissons de forte valeur par les pays en développement seront consommées de manière croissante par ces pays.

Cela n'exclut pas la poursuite de forts échanges mondiaux de produits aquatiques: en 2002, $38 \%$ de la production mondiale totale (alimentaire et non alimentaire), soit environ 50 millions de tonnes en "équivalent produits frais " était tiques alimentaires entre pays développés

exportée sous forme brute (contrairement à la plupart des productions, les produits aquatiques frais ont une valeur généralement supérieure aux produits transformés) et transformée (SOFIA, 2004), avec, en outre, des activités importantes d'import-export pour certains pays : ainsi, la Chine était à la fois le premier exportateur et le huitième importateur mondial, les États-Unis le quatrième exportateur et deuxième importateur (derrière le Japon). Pour les PED, le solde net de ces échanges s'est élevé à $+17,4$ milliards de dollars, venant en première place loin devant les exportations d'autres produits agricoles comme le café, le cacao ou la banane.

Mais, du fait de la forte spécialisation alimentaire des deux blocs, les échanges de produits aquatiques alimentaires se feront pour l'essentiel en leur sein. En revanche, les échanges de produits non alimentaires, en particulier de farines et huiles de poisson demeureront actifs, avec des flux nets d'exportation des PED qui devraient se maintenir aux niveaux actuels, en dépit de la croissance de l'aquaculture dans ces pays (Delgado et al., 2003).

\section{Conclusion}

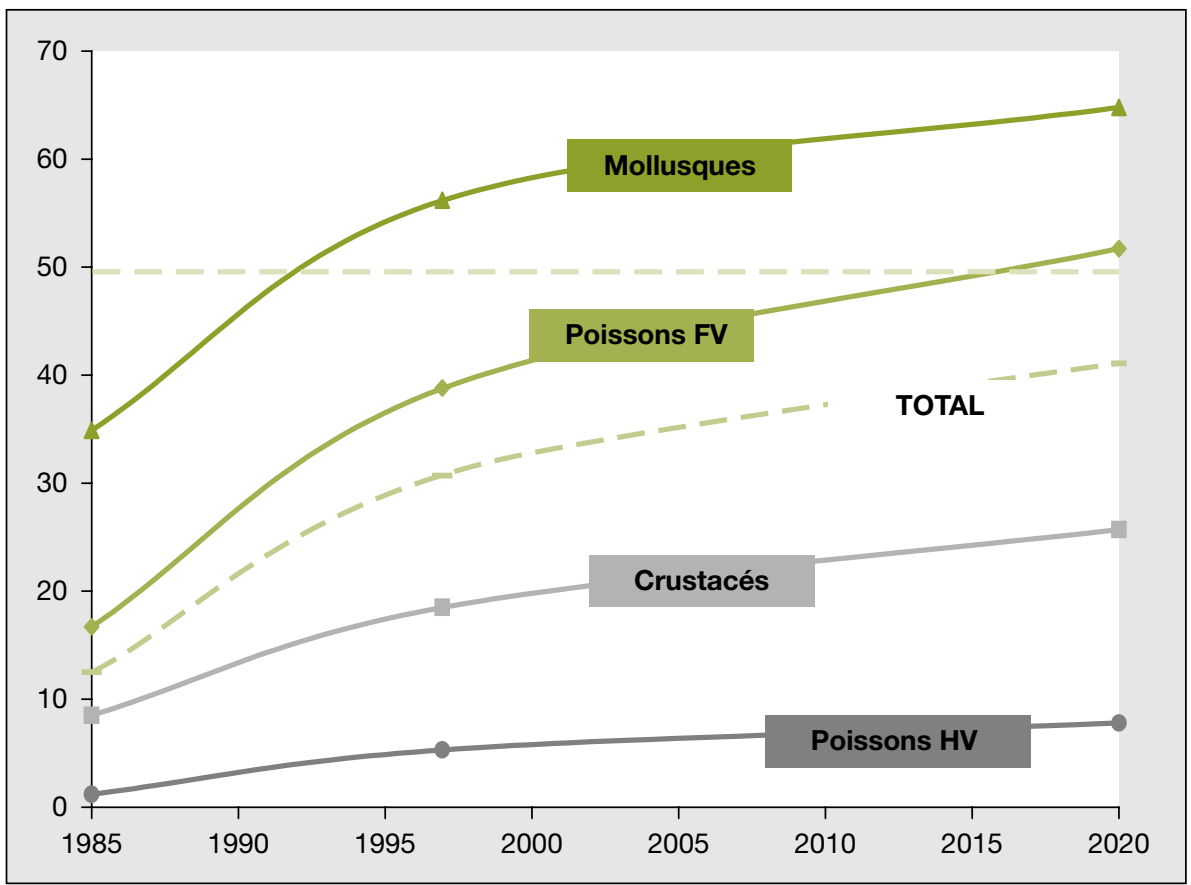

Figure 7. Évolution de la contribution de I'aquaculture en pourcentage à la production mondiale des différents produits aquatiques selon le scénario tendanciel de I'IFPRI (International Food Policy Research Institute).

Figure 7. Variation in aquacultural contribution in percentage to the world production of various aquatic products according to the International Food Policy Research Institute (IFPRI's) trend-based scenario.
La tendance à une augmentation de la demande en protéines animales dans les pays en développement, sous-tendue par une augmentation prévisible des revenus par habitant dans de nombreux pays, devrait se poursuivre.

Le cas des productions aquatiques amène à distinguer nettement la situation des pêcheries de celle de l'aquaculture. Dans le domaine des pêcheries, plusieurs facteurs conduisent à penser que la stabilisation des captures observée depuis le début des années 1990 ne représente pas une situation d'équilibre durable, avec des scénarios d'érosion progressive des captures totales, tant minotières qu'alimentaires, aboutissant, a fortiori, à une réduction substantielle des apports par habitant.

Dans le domaine de l'aquaculture, la dynamique actuelle devrait, en revanche, se poursuivre, avec des taux de croissance sans doute plus modérés, en particulier en Chine, en dépit des contraintes croissantes qui pèseront sur l'utilisation des farines et huiles de poisson en aquaculture intensive. Les taux de croissance des différentes productions devraient 
demeurer assez voisins, ce qui ne devrait pas modifier notablement la répartition géographique et l'importance relative des productions.

L'analyse de l'évolution des échanges de produits aquatiques alimentaires entre pays développés et pays en développement met en évidence que ces flux devraient se restreindre avec une tendance croissante à "l'autoconsommation".

La solidarité intergénérationnelle, dimension majeure du développement durable, appliquée aux ressources aquatiques, concerne aussi bien une réduction délibérée de l'exploitation de certains stocks, afin de permettre leur reconstitution, qu'une diminution des impacts multiples et grandissants sur les milieux aquatiques, dans le but de préserver leur potentiel aquacole.

\section{Références}

Brugère C, Ridler N. Global aquaculture outlook in the next decades: an analysis of national production forecasts to 2030. FAO Fisheries Circular. Rome: FAO, 2004.
Cury $P$, Morand S. Biodiversité marine: une dynamique d'interactions où l'humain est présent. In: Barbault R, Chevassus-au-Louis B, eds. Biodiversité et changements globaux. Enjeux de société et défis pour la recherche. Paris: ADPF ; mministère des Affaires étrangères, 2005 : 50-79.

Delgado C, Rosegrant M, Steinfeld H, Ehui S, Courbois C. L'élevage d'ici 2020: la prochaine révolution alimentaire. SI: IFPRI; FAO; ILRI, 1999.

Delgado C, Wada N, Rosegrant MW, Meijer S, Ahmed M. Fish to 2020. Supply and Demand in Changing Global Markets. SI: IFPRI; WFC, 2003.

De Silva SS, MoehI J, Satia B, Bartley D, Subasinghe R. Aquaculture et pêche dans les eaux intérieures; synergie pour une production durable de poisson destiné à l'alimentation humaine. In: État de l'aquaculture dans le monde. FAO Circulaire sur les pêches $\mathrm{n}^{\circ} 886$, Rev. 2. Rome: FAO, 2003. www.fao.org/documents/

FAO. État de l'aquaculture dans le monde. Rome: FAO, 2003.

FAO. Marine ranching. FAO Fisheries Technical Paper. Rome: FAO, 2004.

FAOSTAT. http://faostat.fao.org/site/565/ default.aspx.

IFFO. 2007: http://www.iffo.net.

Jackson JBC, Kirby M, Berger WH, et al. Historical overfishing and the recent collapse of coastal ecosystems. Science 2001; 293: 629-38.
Li SF. Aquaculture research and its relation to development in China. In : Zhang LX, Liu J, Li SF, Yang NS, Gardiner PR, eds. Agricultural development and the opportunities for aquatic resources research in China. Penang: WorldFish Center, 2002.

New M, Wijkström U. Use of fishmeal and fish oil in aquafeeds: further thoughts of the fishmeal trap. Rome: FAO, 2002.

Pike IH. Eco-efficiency in aquaculture global catch of wild fish used in aquaculture. Int Aquafeed $2005 ; 8$ : 38-40.

Salvanes AGV. Ocean Ranching. In: Steele J, Turekian KK, Thorpe SA, eds. Encyclopedia of Ocean Sciences. $\mathrm{N} \times$ 4. London : Academic Press, 2001. www.bio.uib.no/biodoc/pdffiles/ pub/1326.pdf.

SOFIA. The State of world Fisheries and Aquaculture. Rome: FAO, 2002.

SOFIA. The State of world Fisheries and Aquaculture. Rome: FAO, 2004.

SOFIA. The State of world Fisheries and Aquaculture. Rome: FAO, 2006.

Tacon AGC, Hasan MR, Subasinghe RP. Use of fishery resources as feed inputs to aquaculture development: trends and policy implications. FAO Fisheries Circular $n^{\circ}$ 1018. Rome: FAO 2006.

Wijkström U. Short and long-term prospects for consumption of fish. Vet Res Commun 2003 ; 27 (Suppl. 1) : 461-8.

$\mathrm{Ye} \mathrm{Y}$. Historical consumption and future demand for fish and fishery products: exploratory calculations for the years 2015-2030. Rome: FAO, 1999. 